\title{
1 CRYOBIOLOGY OF CEPHALOPOD (Illex coindetti) SPERMATOPHORES
}

2 Vanesa Robles ${ }^{1,2 * \#}$, Felipe Martínez-Pastor ${ }^{1,2} *$, Giuliano Petroni ${ }^{3}$, Marta F. Riesco ${ }^{1,2}$,

3 Anna Bozzano ${ }^{3}$, Roger Villanueva ${ }^{3}$

4 *Authors have equally contributed to the work

$5{ }^{1}$ Department of Molecular Biology, Faculty of Biology, University of León, 24071,

6 Spain

$7 \quad 2$ INDEGSAL, University of León, 24071, Spain

$8{ }^{3}$ Institut de Ciències del Mar (CSIC), Passeig Maritim de la Barceloneta 37-49, 08003-

9 Barcelona, Spain

10 \#corresponding author: Vanesa Robles, Tel. (+34) 987291000 ext 5678 email

11 v.robles@unileon.es

12

13

14 


\section{Abstract}

17 Cephalopod culture is expected to increase in the near future and sperm 18 cryopreservation would be a valuable tool to guarantee sperm availability throughout 19 the year and to improve artificial insemination programs. We have studied the tolerance 20 of spermatophores from the oceanic squid Illex coindetii to several cryoprotectants, in 21 two toxicity experiments and a cryopreservation test. Five permeating cryoprotectants 22 were tested: Dimethyl sulfoxide (Me2SO), methanol, glycerol, propylene glycol and 23 ethylene glycol. In the first experiment, spermatophores were exposed to the five 24 cryoprotectants at $5 \%(\mathrm{v} / \mathrm{v})$ and $15 \%(\mathrm{v} / \mathrm{v})$ at $4{ }^{\circ} \mathrm{C}$ for $5 \mathrm{~min}$. In the second experiment,

25 spermatophores were exposed to the cryoprotectants at $15 \%$ using different exposure 26 times: 5, 15 and $30 \mathrm{~min}$. In a third experiment, we tested two cryopreservation 27 protocols: $\mathrm{LN}_{2}$ vapor or $-80{ }^{\circ} \mathrm{C}$ freezer, using a $15 \%$ cryoprotectant and 15 or 30 min of 28 exposure. Viability and mitochondrial activity were assessed using Mitotracker deep 29 red, YOPRO1 and Hoechst 33342, by flow cytometry. Spermatozoa in this species 30 remain viable after cryoprotectant exposure but their quality decreased considerably

31 after cryopreservation, only $5 \%$ to $10 \%$ of spermatozoa being motile. Flow cytometry

32 demonstrated that Me2SO may be the most appropriate cryoprotectant for I. coindetii 33 spermatozoa, and shows a first approach on cephalopod sperm cryopreservation, 34 opening new possibilities for the research and culture of this group of molluscs.

36 Keywords: Mollusca, spermatophore, spermatangia, spermatozoa, cryopreservation, 37 flow cytometry. 


\section{1. Introduction}

Cephalopods are a group of marine molluscs of great importance not only for the

41 seafood industry, but also for the biomedical, pharmaceutical and cosmetic industries.

42 They are mainly obtained by fishing, whereas their aquaculture production is still very 43 limited: 3,652,632 tons by fishing versus 10 tons by aquaculture during 2010 [10].

44 Nevertheless, cephalopod aquaculture production is expected to increase in the near

45 future attending to recent scientific advances [8,23,35,37]. The expansion of cephalopod

46 breeding will require advances in reproductive management and the development of

47 reproduction technologies.

48 In particular, sperm cryopreservation is a technique that allows spermatozoa to

49 be stored indefinitely. Among the many advantages for breeding programs, it allows

50 sperm availability throughout the year (especially important in seasonal species, or

51 when gamete production differs among sexes or is unpredictable), and a more efficient

52 management of fertilization and selection programs [40]. Specifically, artificial

53 insemination programs would benefit from sperm cryopreservation, improving the

54 culture of oceanic squid species by using in vitro fertilization [38,41]. These

55 technologies could also be used for the preservation of endangered cephalopod species

56 such as Nautilus pompilius [9].

57 In cephalopods, spermatozoa can be collected either from mature males or from

58 copulated females. Males produce spermatophores that are stored in the spermatophoric

59 organ (Needham's sac). Copulated females have ejaculated spermatophores called 60 spermatangia anchored to their bodies. Spermatozoa from spermatophores and

61 spermatangia have similar fertilizing capacity and fertilization rates [24].

62 Studies have been carried out on spermatozoon structure and morphological

63 characteristics in several species of cephalopods [5,7,13,14,16,17,25,27], spermatophore 64 physics, morphology and physiology [2,33], and spermatangium characteristics $65[19,20,21,32]$. Nevertheless, very little information on spermatophores or 66 spermatangium is available [22]. As far as we know, the only precedent on cephalopod 
67 spermatophore refrigeration was reported by Naud \& Havehand [31] for the cuttlefish

68 Sepia apama and no published information exists on sperm, spermatophore or

69 spermatangium cryobiology for any cephalopod species.

In this study, we used the broadtail shortfin squid Illex coindetii (Vérany, 1839),

71 which is a medium-sized oceanic species widely-distributed on both sides of the

72 Atlantic Ocean and in the Mediterranean Sea. This species belongs to the

73 Ommasthephidae family ("flying squid"), which includes many species worldwide,

74 including many of great commercial importance (half of the world's squid captures

75 correspond to one species of this family, Dosidicus gigas) Illex coindetii is captured

76 throughout the year, mostly from bottom and pelagic trawls, and has a high commercial

77 interest [35]. I. coindetii males produce a mean of 465 spermatophores, with lengths

78 ranging from 11 to $38 \mathrm{~mm}$, the length being proportional to male size $[15,18]$.

79 Copulated females have a mean of 484 spermatangia (13 $\mathrm{mm}$ mean length), attached in

801 to 6 bulbs on the internal mantle cavity, at the base of the gills [18]. Images of a

81 spermatophore and spermatangia of Illex coindetii are shown in Figure 1, while further

82 information can be found in Nigmatullin et al., 2003 [33].

83 It is well known that the use of cryoprotectants is required to ensure proper cell

84 protection during the cryopreservation process. However, these agents can be toxic to

85 spermatozoa, and the evaluation of such effects should be carefully studied before

86 designing any cryopreservation protocol [36]. Moreover, the equilibration time in a

87 solution with cryoprotectants should be long enough to allow the cryoprotectant to

88 interact with the cells while minimizing toxic effects. Permeating cryoprotectants exert

89 their protective effects by entering the cell, and require some time to permeate the

90 plasma membrane and equilibrate with the external concentration, depending on its

91 chemical structure and temperature. Furthermore, the cell must also undergo osmotic

92 changes while the cryoprotectant enters and equilibrates [26], and recover from them.

93 Moreover, the cryopreservation protocol (cooling and thawing rate, cooling and freezing

94 method, container size and shape, etc.), critically affects post-thawing sperm viability, 
95 and it could interact with the effects of the cryoprotectant, either in a positive or 96 detrimental way.

97 In this study, we carried out several toxicity experiments in an attempt to collect

98 basic data on the tolerance of from $I$. coindetii spermatozoa to several permeating

99 cryoprotectants, including a preliminary cryopreservation trial to investigate post-

100 thawing sperm quality after using selected cryoprotectant protocols. In this species, the

101 manipulation of spermatophores is easier than free spermatozoa, therefore we used

102 whole spermathophores as the experimental units for the toxicity and cryopreservation

103 trials, rather than free spermatozoa.

104

\section{2. Materials and Methods}

1062.1 Animals and samples

107 Mature individuals of the broadtail shortfin squid, Illex coindetii, were captured

108 by the local bottom-trawl fleet in the Mediterranean near Barcelona, Spain, (April to 109 September, 2010). Whole squid were transported in ice to the laboratory. 110 Spermatophores were collected by dissecting the spermatophoric organ (Needham'sac)

111 of mature males measuring from $107 \mathrm{~mm}$ to $163 \mathrm{~mm}$ (mantle length) and weighing 112 from $48 \mathrm{~g}$ to $158 \mathrm{~g}$. Spermatangia were collected from copulated females measuring 113 from $152 \mathrm{~mm}$ to $198 \mathrm{~mm}$ (mantle length) and weighing from $100 \mathrm{~g}$ to $196 \mathrm{~g}$. Bulbs of

114 spermatangia were dissected from the females using scissors, placed on a 1-mm mesh 115 and vigorously flushed with seawater to remove organic debris from the surface. Three 116 to four hours after squid collection at sea, spermatophores and spermatangia were 117 individually placed in $5 \mathrm{ml}$ plastic containers, covered with $0.2-\mu \mathrm{m}$ filtered sea water 118 (FSW) and stored at $4{ }^{\circ} \mathrm{C}$ for $12-14 \mathrm{~h}$ before being sent to the University of León at the 119 same temperature. Samples were processed approximately $48 \mathrm{~h}$ after squid collection at 120 sea.

\section{$122 \quad 2.2$ Scanning electron microscopy of Illex spermatozoa}


124 small portions ( $<2 \mathrm{~mm}$ length) using scissors. To promote sperm activation, the chopped

125 sperm mass from groups of 3-4 bulbs of spermatangia was added to a glass container

126 with $10 \mathrm{ml}$ of FSW and gently shaken. The milky solution was filtered through a 100-

$127 \mu \mathrm{m}$ mesh to remove spermatangia capsule debris. Cover glasses were submerged in the

128 milky solution to be impregnated by sperm, then fixed in $2.5 \%$ glutaraldehyde in FSW

129 for $15 \mathrm{~h}$, washed in FSW, followed by dehydration in an increasing concentration (v/v)

130 of ethanol $(20 \%, 30 \%$ and $50 \%)$ and stored in ethanol $70 \%$ at $4{ }^{\circ} \mathrm{C}$. At the beginning of

131 the SEM preparation, the samples were again dehydrated in an increasing concentration

132 of ethanol $(80 \%, 90 \%$, and $95 \%)$ until saturated in absolute ethanol. Each ethanol bath

133 lasted $10 \mathrm{~min}$. After complete dehydration in the ethanol series, the samples were dried

134 to the critical point in a Bal-Tec CPD 030 Drier using $\mathrm{CO}_{2}$ as the transition liquid. After

135 the drying stage, the samples were mounted on stubs with double-sided conductive

136 sticky tape to orientate them in the preferred position. The mounted samples were

137 sputter coated with gold-palladium in a Polaron Sputter Coater SC500 and then

138 observed using a scanning electron Hitachi S3500N microscope with working voltages

139 of $5 \mathrm{kV}$. Measurements of spermatozoa were obtained using the Image-Pro Plus 5.0

140 image analyser.

$142 \quad 2.3$ Experimental design

143 2.3.1 Experiment 1: Toxicity study comparing different concentrations of 144 cryoprotectants

145 Five permeating cryoprotectants were used for toxicity studies: Me2SO, methanol, 146 glycerol, propylene glycol and ethylene glycol. Spermatophores were exposed to each 147 cryoprotectant $(5 \%$ and $15 \%(\mathrm{v} / \mathrm{v})$ in $\mathrm{FSW})$ at $4{ }^{\circ} \mathrm{C}$. Incubation in $\mathrm{FSW}$ without 
148 cryoprotectant was used as a control. After $5 \mathrm{~min}$, the spermatophores were passed to 149 two dilutions (1/2 and 1/4) with FSW, and finally placed in pure FSW. Each washing 150 step lasted $2 \mathrm{~min}$. After the third washing step, the spermatophores were placed on a 151 glass slide and dissected using fine forceps. The viability and mitochondrial activity of 152 the sperm mass was evaluated immediately by flow cytometry. Spermatophores from 153 six males were used in this experiment.

155 2.3.2 Experiment 2: Toxicity study comparing different exposure times to 156 cryoprotectants

157 The same five permeating cryoprotectants were used (Me2SO, methanol, glycerol, 158 propylene glycol and ethylene glycol) at $15 \%(\mathrm{v} / \mathrm{v})$. Spermatophores were exposed to 159 the cryoprotectant at $4{ }^{\circ} \mathrm{C}$ for 3 times: 5, 15 and 30 min. Incubation in FSW without 160 cryoprotectant was used as a control. The spermatophores were then washed in two 161 progressive dilutions of FSW (1/2 and 1/4), and finally placed in pure FSW. Each 162 washing step lasted $2 \mathrm{~min}$. After the third washing step, the spermatophores were 163 assessed as in Experiment 1. Spermatophores from ten males were used in this 164 experiment.

166 2.3.3 Experiment 3: Cryopreservation of spermatophores (liquid nitrogen vapor vs. 167 cryopreservation at $-80^{\circ} \mathrm{C}$ )

168 Spermatophores were loaded into cryovials containing $1 \mathrm{~mL}$ of FSW with each 169 of the five permeating cryoprotectants (Me2SO, methanol, glycerol, propylene glycol 170 and ethylene glycol) at $15 \%(\mathrm{v} / \mathrm{v})$. Two trials were performed. In the first one, the 171 spermatophores were exposed to the cryoprotectant at $4{ }^{\circ} \mathrm{C}$ for $15 \mathrm{~min}$ or $30 \mathrm{~min}$, and 172 then frozen using $\mathrm{LN}_{2}$ vapors, as described later. In the second one, the spermatophores 173 were exposed to the cryoprotectant at $4{ }^{\circ} \mathrm{C}$ for $15 \mathrm{~min}$. The samples were then frozen 174 using either: (1) $\mathrm{LN}_{2}$ vapors or (2) cryopreservation at $-80{ }^{\circ} \mathrm{C}$. In the first method $\left(\mathrm{LN}_{2}\right)$, 175 cryovials were placed $1 \mathrm{~cm}$ above $\mathrm{LN}_{2}$ inside a closed styrofoam box. After $30 \mathrm{~min}$, the 
176 cryovials were immersed in $\mathrm{LN}_{2}$ and stored in cryoboxes in Dewar tanks containing $177 \mathrm{LN}_{2}$. The freezing rate was $-15{ }^{\circ} \mathrm{C} / \mathrm{min}$ from $4{ }^{\circ} \mathrm{C}$ to $-20{ }^{\circ} \mathrm{C}$ and $-51{ }^{\circ} \mathrm{C} / \mathrm{min}$ from $-20{ }^{\circ} \mathrm{C}$ 178 to $100{ }^{\circ} \mathrm{C}$ (determined in previous studies) [4]. In the second method (freezing at $17980^{\circ} \mathrm{C}$ ), cryovials were put in a cryo-freeze container (Nalgene, Denmark), which was 180 placed in a $-80{ }^{\circ} \mathrm{C}$ freezer providing a $-1{ }^{\circ} \mathrm{C} / \mathrm{min}$ cooling rate (manufacturer's

181 specifications). Samples frozen at $-80{ }^{\circ} \mathrm{C}$ were stored at that temperature.

182 Thawing was carried out after one week. The cryovials were immersed in a $30{ }^{\circ} \mathrm{C}$ water 183 bath for $2 \mathrm{~min} 20 \mathrm{~s}$. After thawing, the spermatophores were washed and dissected as 184 described for experiments 1 and 2. Viability and mitochondrial activity of the sperm 185 mass were assessed immediately. Spermatophores from eight males were used in this 186 experiment. For freezing in $\mathrm{LN}_{2}$ vapor after $30 \mathrm{~min}$ of exposure to cryoprotectant and 187 for freezing at $-80{ }^{\circ} \mathrm{C}$, experiment was performed in triplicate.

\section{$190 \quad 2.4$ Evaluation of sperm viability and mitochondrial activity}

191 Sperm viability (plasma membrane integrity) and mitochondrial activity were

192 evaluated using fluorescent probes and flow cytometry. After extracting the sperm mass, 193 it was diluted with $50 \mu 1$ FSW added on the slide. Twenty-five microliters were added to $194300 \mu 1$ of FSW in a polypropylene tube, with $100 \mathrm{nM}$ Mitotracker deep red (MT), $195100 \mathrm{nM}$ YOPRO1 and $5 \mu \mathrm{M}$ Hoechst 33342 (H342). The MT and YOPRO1 were 196 purchased from Invitrogen (Barcelona, Spain) and the H342 from Sigma (Madrid, 197 Spain). Our group has successfully tested these stains in fish (unpublished data) and 198 mammalian spermatozoa [28]. Mitotracker deep red (MT) accumulates into 199 mitochondria with high membrane potential, thus discriminating cells with active 200 mitochondria. YOPRO1 can penetrate cells with increased membrane permeability, 201 intercalating into DNA and thus staining the nuclei of these cells. Hoechst 33342 202 (H342) is known to permeate the membranes of mammalian spermatozoa, staining the 203 nuclei of all spermatozoa [30]. However, in the present study we found that this dye 
204 could not enter squid spermatozoa with intact membranes (YOPRO1-) and only stained 205 part of the YOPRO1+ subpopulation. Therefore, it seems that H342 stains squid 206 spermatozoa when membranes are damaged. YOPRO1+/H342- spermatozoa were 207 considered membrane-intact, but with increased permeability, whereas YOPRO$2081+/ \mathrm{H} 342+$ spermatozoa were considered membrane-damaged.

209 After 5 min at ambient temperature, the sample was analyzed by flow cytometry (CyAn 210 ADP, Beckman Coulter. Fullerton, CA, USA), carrying out a multicolor experiment. 211 H342 was excited by a violet laser $(405 \mathrm{~nm})$ and the emission collected using a $212450 / 50 \mathrm{~nm}$ filter; YOPRO1 was excited by a blue laser $(488 \mathrm{~nm})$ and the emission 213 collected using a 530/40 nm filter; Mitotracker deep red was excited by a red laser $214(635 \mathrm{~nm})$ and the emission collected using a 665/20 nm filter. Events were first plotted 215 in a forward scatter vs. sideward scatter plot, and a gate was defined around the cloud of 216 events corresponding to spermatozoa (validated using $\mathrm{H} 342+$ events, unequivocally 217 identified with spermatozoa). Only events falling in that gate were considered as 218 spermatozoa for fluorescence analysis. The fluorochrome combination allowed us to 219 distinguish four subpopulations of spermatozoa: H342-/YOPRO1- were considered 220 membrane-intact (viable) spermatozoa; H342-/YOPRO1+ were considered

221 spermatozoa with increased membrane permeability; H342-/YOPRO1-/MT+ were 222 considered viable spermatozoa with intact membranes that also had active 223 mitochondria, and were expressed as the ratio of YOPRO1-/MT+ within YOPRO1224 (membrane intact) spermatozoa; H342+ spermatozoa were considered non-viable 225 (damaged membranes). Ten thousand events were read per sample.

\subsection{Evaluation of sperm motility}

228 On some occasions (this could not be performed systematically), sperm motility 229 was subjectively checked after activating the spermatozoa with FSW. Motility was 230 observed at room temperature using a Nikon E800 equipped with a $\times 10$ objective and 231 phase contrast optics. 


\section{2.6 Statistical analysis}

234 The statistical analyses were performed using the R statistical environment [6]. Viability

235 and mitochondrial activity (as a \% of each sperm subpopulation) were analyzed using 236 linear mixed-effects models, with cryoprotectant, concentration, exposure time or 237 cryopreservation method as fixed effects (depending on the experiment), and using the 238 male as the grouping factor for the random part of the model. When required pairwise 239 comparisons, were carried out using Tukey correction. Results are shown as mean \pm 240 SEM, unless otherwise specified.

\section{3. Results}

\section{3.1 Scanning electron microscopy of Illex spermatozoa}

244 Observations from scanning electronic microscopy showed that Illex coindetii 245 spermatozoa have a cylindrical-shaped head and two tails (Figure 2). Thirteen 246 spermatozoa were measured, presenting mean \pm SD values. The whole head region

247 (acrosome + nucleus + nuclear appendage or mitochondrial spur) measured $6.83 \pm$ $2481.39 \mu \mathrm{m}$ long $(\mathrm{n}=13)$. The acrosome is a tronco-conical structure $0.43 \pm 0.17 \mu \mathrm{m}$ long 249 positioned at the apex of the nucleus. The nucleus is elongated $(5.12 \pm 1.25 \mu \mathrm{m}$ long, $2501.39 \pm 0.08 \mu \mathrm{m}$ wide), being broadest at three-quarters of its length in correspondence 251 with the annular atria (the insertion of the tails into the nucleus-spur border), a funnel252 like structure which the long flagella projects. A cone-shaped appendage $(1.41 \pm$ $2530.60 \mu \mathrm{m}$ long) projects behind the posterior part of the nucleus. The tails were $53.22 \pm$ $25411.50 \mu \mathrm{m}$ long and $0.24 \pm 0.05 \mu \mathrm{m}$ wide $(\mathrm{n}=22)$. The structure became more 255 filamentous in the distal part of the tail. The total length of the spermatozoon was $256 \quad 58.49 \pm 4.40 \mu \mathrm{m}(\mathrm{n}=22)$. 


\subsection{Flow cytometry analyses of fresh spermatoza}

The flow cytometry analyses yielded defined populations according to previous studies in other species, showing high repeatability [30]. Flow cytometry assays of the untreated spermatophores showed that a large proportion of spermatozoa were membrane-intact $(57.2 \% \pm 6.1)$, with a minor population of spermatozoa showing increased membrane permeability $(18.5 \% \pm 2.1)$. Almost all spermatozoa with intact membranes also had active mitochondria $(95.0 \% \pm 0.7$ of YOPRO1-- spermatozoa).

\subsection{Results of the toxicity assays}

In Experiment 1, toxicity assays comparing $5 \%$ and $15 \%$ of the five cryoprotectants showed minor differences among cryoprotectants or concentrations (Figure 3). Only the concentration, as a main effect, significantly affected the proportion of spermatozoa with intact membranes $(\mathrm{P}<0.001)$ and damaged membranes $(\mathrm{P}=0.011)$, but neither the cryoprotectant type as a main effect nor the interaction of concentration $\times$ cryoprotectant type were significant. Subjecting the spermatophores to $5 \%$ cryoprotectant resulted in a slight decrease in membrane integrity (intact membrane: $48.9 \% \pm 3.6$; damaged membrane: $26.2 \% \pm 3.4$ ), which was significant in comparison with the control (intact membrane: $57.2 \% \pm 6.1, \mathrm{P}<0.001$; damaged membrane: $24.3 \% \pm$ 5.6, $\mathrm{P}=0.018$ ), or with $15 \%$ (intact membrane: $54.9 \% \pm 3.1, \mathrm{P}<0.001$; damaged membrane: $24.0 \% \pm 2.5, \mathrm{P}=0.003)$. The effect of the concentration was also significant for the proportion of spermatozoa with increased membrane permeability and with active mitochondria, but in this case, we detected a significant interaction between the concentration and the cryoprotectant. Therefore, we performed individual comparisons among treatments. A comparison within each cryoprotectant showed that the proportion of spermatozoa with intact membranes was higher for $5 \%$ methanol $(31.5 \% \pm 6.2)$ and $5 \%$ ethylene glycol $(28.2 \% \pm 5.4)$ than for the control $(18.5 \% \pm 2.1)$ and $15 \%$ ethylene glycol $(13.7 \% \pm 3.1)$, respectively, with no other significant differences found. The proportion of spermatozoa with active mitochondria (within membrane-intact spermatoza) was lower in $5 \%$ methanol $(86.0 \% \pm 3.5), 15 \%$ glycerol $(90.8 \% \pm 2.5)$ and 
$28515 \% \mathrm{Me} 2 \mathrm{SO}(87.4 \% \pm 3.2)$ in comparison with comparing with the control. Similarly, $28615 \%$ Me2SO was significantly lower than the control and 5\% Me2SO $(96.1 \% \pm 0.3$

287 overall). Nevertheless, we have to consider the higher viability of samples frozen with $28815 \% \mathrm{Me} 2 \mathrm{SO}$, and therefore when considering the absolute proportions of spermatozoa 289 with active mitochondria the differences were not significant $(5 \%: 48.2 \% \pm 3.4 ; 15 \%$ : $29042.0 \% \pm 2.2 ; \mathrm{P}>0.05)$. Taking into account the results of Experiment 1, there were few 291 differences between the two concentrations. Therefore we selected the $15 \%$ 292 concentration for carrying out Experiment 2. The time-dependent variation of the 293 membrane and mitochondrial status for each cryoprotectant are shown in the Figure 4. 294 There were few differences among cryoprotectants, and membrane status was not 295 significantly affected by exposure time (although there was a trend towards increasing 296 membrane damage with time). However, glycerol yielded a lower proportion of 297 spermatozoa with intact membranes $(\mathrm{P}<0.05$ comparing with $\mathrm{Me} 2 \mathrm{SO}$ or ethylene 298 glycol) and a higher proportion of spermatozoa with damaged membranes $(\mathrm{P}<0.05$ 299 comparing with ethylene glycol at $5 \mathrm{~min} ; \mathrm{P}<0.01$ comparing with ethylene glycol and 300 methanol; $\mathrm{P}<0.001$ comparing with $\mathrm{ME2SO}$ at $15 \mathrm{~min}$ ). At $30 \mathrm{~min}$, glycerol yielded the 301 highest proportion of membrane damage $(40.5 \% \pm 9.7)$, although differences were not 302 significant due to an increase in within-replicate variability at that time. Nevertheless, 303 models showed an interaction of cryoprotectant and time for glycerol, indicating a 304 significant decrease in sperm viability with time when using this cryoprotectant. Both 305 membrane permeability and mitochondrial activity were significantly affected by the 306 incubation time, whereas the cryoprotectant type did not show significant effects. Thus,

307 the population of spermatozoa with increased membrane permeability grew from $21.1 \%$ $308 \pm 1.6$ at $5 \mathrm{~min}$ to $34.7 \% \pm 2.3$ at $30 \mathrm{~min}(\mathrm{P}=0.021)$. Likewise, almost all spermatozoa 309 with intact membranes showed active mitochondria at $5 \mathrm{~min}(91.0 \% \pm 1.3)$, this 310 proportion lowering to $43.5 \% \pm 5.7$ at $30 \min (\mathrm{P}=0.007)$.

\subsection{Results of the cryopreservation trials}


312 In Experiment 3, we tested the suitability of the cryoprotectants for freezing the

313 spermatophores. Despite the good membrane and mitochondrial status of the 314 spermatozoa in previous experiments, quality decreased considerably after

315 cryopreservation using $\mathrm{LN}_{2}$ vapours, with membrane integrity and mitochondrial status 316 dropping almost to $0 \%$ in most treatments. Only $15 \% \mathrm{Me} 2 \mathrm{SO}$, either with 15 or $30 \mathrm{~min}$

317 of incubation before freezing, showed some ability to preserve membrane integrity [5\% $318 \pm 1.5$ vs. $1.5 \% \pm 0.5$ (pooled results from the other cryoprotectants); $\mathrm{P}<0.001$ ]. $\mathrm{Me} 2 \mathrm{SO}$

319 also achieved a higher percentage of spermatozoa maintaining active mitochondria $320(9.4 \% \pm 1.6$ vs. $4.4 \% \pm 0.7 ; \mathrm{P}<0.01)$ and a lower percentage of membrane-damaged 321 spermatozoa $(85.6 \% \pm 2.8$ vs. $94.1 \% \pm 1.1 ; \mathrm{P}<0.001)$. Using a freezer $\left(-80{ }^{\circ} \mathrm{C}\right)$ for 322 freezing the cryovials resulted in less than $1 \%$ viable spermatozoa in all treatments. 323 Motility was checked in several samples, and only those frozen with Me2SO and with $324 \mathrm{LN}_{2}$ vapours maintained a low proportion of spermatozoa capable to swim after 325 freezing-thawing and activation (5\% to $10 \%$ of motile spermatozoa).

326 Moreover, freezing/thawing seems to damage the spermatophore. We could 327 observe signs of wear on the spermatophore, and spontaneous spermatophoric reaction 328 when washing the thawed spermatophores. Nevertheless, these observations seemed 329 unrelated to the quality of spermatozoa (data not shown).

\section{4. Discussion}

332 This is the first attempt to explore conditions for spermatophore 333 cryopreservation in Cephalopoda. The interest of this study lies in the fact that some 334 species within this class could be cultured in the near future, and thus the availability of 335 cryopreservation methods for long-term sperm storage would be a valuable tool for 336 farms and to maintain diversity in farmed stocks, or to select and breed desirable traits. 337 Whereas no information is available for cephalopods, there are reports of successful 338 cryopreservation of spermatozoa and spermatophores in other marine invertebrates. As 339 an example, spermatophores and spermatozoa have been cryopreserved in the mud crab 
340 (Scylla serrata) [3], in the black tiger shrimp (Penaeus monodon) [34,39] and in the 341 giant freshwater prawn (Macrobrachium rosenbergii) [1]. For practical reasons, we have

342 only used whole spermatophores or spermatangia, rather than the free sperm mass. The 343 possibility of cryopreserving whole spermatophores offers several advantages, since 344 manipulation is easier and the spermatophores are structures whose function is to 345 protect spermatozoa from stress, and to maintain them in a quiescent state. However, 346 since spermatophores isolate spermatozoa from the environment, they might interfere 347 with the cryopreservation process (delaying slowing cryoprotectant equilibration, for 348 instance). We did not perform a comparison by using free spermatozoa in the three 349 experiments, so this issue remains unsolved for now.

$350 \quad$ This study also presents a working flow cytometry protocol for analyzing squid 351 spermatozoa, which allows the membrane and mitochondrial status of squid 352 spermatozoa to be assessed (adapted from published protocols in other species [30]). 353 Interestingly, Hoechst 33342, which stains the whole sperm population in other species 354 [28], could not permeate the membrane of squid spermatozoa (we cannot exclude the 355 presence of membrane transporters causing Hoechst 33342 excursion), therefore 356 identifying a disrupted-membrane sperm subpopulation.

357 Our results show that untreated spermatophores, despite being refrigerated for 358 two days, had a high proportion of membrane intact spermatozoa (57.2\%, SD: 15.0$)$.

359 These data suggest that spermatophores can be successfully transported in FSW at $4{ }^{\circ} \mathrm{C}$ 360 and conserved for up to $48 \mathrm{~h}$ at least before being processed, although further 361 experiments are necessary. Experiments in crustaceans have showed that refrigerated 362 spermatophores can maintain high sperm viability for weeks and even a month and 363 Naud \& Havehand [31] reported that in spermatophores of the cuttlefish Sepia apama 364 stored at $4{ }^{\circ} \mathrm{C}$ motility was still observed in resuspended

365 sperm after two months.

366 One of the most important steps in designing a cryopreservation protocol is the 367 selection of a proper cryoprotectant combination as well as the optimum concentrations 
368 and exposure times [11]. Although cryoprotectants prevent cell damage during 369 freezing/thawing, they are usually toxic [36]. In our study we have found that, when

370 applied to spermatophores/a, cryoprotectant exposure exerts few negative effects on

371 Illex spermatozoa. We have to take into account that both the addition and removal of 372 permeating cryoprotectants subject the spermatophore and the spermatozoa to fast 373 osmotic shocks [12], which could affect the protective role of the former and the post374 thawing viability of the latter.

375 It could be argued that the spermatophores/a may hamper the entrance of 376 cryoprotectants, therefore limiting their toxic effects on spermatozoa. However, in our 377 time exposure experiment we used an equilibration time of up to $30 \mathrm{~min}$, which is fairly 378 long. Indeed, we detected some negative effects on membrane permeability and 379 mitochondria with time, which could possibly be related (since the mitochondrial 380 function would affect membrane permeability [29]). Interestingly, glycerol caused a 381 higher increase in membrane damage, but no differences were observed in 382 mitochondrial status or membrane permeability in comparison with the other 383 cryoprotectants.

384 Our results suggest that Illex spermatozoa might be resistant to osmotic insults or to 385 other toxic effects of the cryoprotectants. However, we must be aware of the limitations 386 of our experiments. Moreover, we still have to determine the role of the spermatophore 387 as a barrier for cryoprotectants. Replicating our experiments in released spermatozoa 388 (more complex, though, due to the different procedures for washing free cells) would 389 allow us to estimate the "real" resistance of squid spermatozoa to cryoprotectants.

390 Nevertheless, the results we obtained could be useful for designing future 391 experiments. The toxicity results alone highlight glycerol as the least suitable option. 392 They also indicate that cryoprotectant concentrations around 5\% would be less effective 393 (at least when the spermatozoa are kept within the spermatophore) suggesting that a 394 successful protocol should use higher concentrations, while combining this higher 
395 concentration with a relatively short exposure time (better results when using $15 \%$ and $3965 \mathrm{~min})$.

397 Unfortunately, our cryopreservation trial was not useful for confirming our 398 previous findings, due to the extremely low viability obtained after thawing. 399 Nevertheless, this experiment suggests that Me2SO would be the most promising 400 cryoprotectant of those tested in this work. As previously stated, although there are 401 practical reasons for cryopreserving whole spermatophores (easy manipulation), future 402 experiments might be aimed not only at improving the cryopreservation protocol, but 403 also at freezing free spermatozoa. The use of a cryoprotectant could not only be more 404 effective, but it would also enable new approaches such as using straws instead of 405 cryovials to be tried out, allowing faster and more uniform cooling of the sample. In 406 fact, a shortcoming of our experimental design was that we only assayed one thawing 407 speed $\left(30^{\circ} \mathrm{C}\right.$ for $\left.2 \mathrm{~min} 20 \mathrm{~s}\right)$. We chose this thawing protocol because it is described in 408 the bibliography together with a variety of freezing protocols. However, it is true that 409 the thawing protocol should be determined by the freezing protocol (especially 410 regarding the speed of heat exchange). Therefore, we cannot discard that our post411 thawing results are associated to the use of an inappropriate thawing protocol.

412 In conclusion, this study provides the first data on the toxic effects of 413 cryoprotectants used in cephalopod spermatozoa, including a very preliminary 414 cryopreservation trial. Illex spermatozoa displayed few signs of toxicity when exposed 415 to cryoprotectants, but the attempts at cryopreservation were unsuccessful. The toxicity 416 results showed that glycerol might be inadequate for Illex spermatozoa, whereas Me2SO 417 seemed to be the most adequate, especially at $15 \%$ and after an exposure treatment of $4185 \mathrm{~min}$. We have also presented a method to assess membrane and mitochondrial status in 419 cephalopod spermatozoa by using flow cytometry, which was fast and effective. 420 Although this study was limited and our results must be considered with caution, it 421 might help to design future experiments aimed at achieving sperm cryopreservation in 422 Cephalopoda. Freezing free spermatozoa instead of the whole spermatophore should be 
423 attempted in order to better understand the cryobiology of squid sperm, although the

424 convenience of freezing whole spermatophora remains.

5. Acknowledgments Vanesa Robles and Felipe Martínez-Pastor were supported by the

427 Ramón y Cajal program (RYC-2008-02339 and RYC-2008-02560), and Marta F. Riesco

428 was supported by a Junta de Castilla y León PhD grant (European Social Fund). We

429 thank the Spanish Ministry of Science and Innovation (MICINN) (research projects

430 AGL2009-11546 and AGL2009-06994) and the Fundación Ramón Areces, and Cintia

431 Miranda for her technical support. We gratefully acknowledge Mr. J.M. Fortuño (Institut

432 de Ciències del Mar) for assistance and advice in obtaining SEM images.

\section{6. References}

435 1. K. Akarasanon, P. Damrongphol, W. Poolsanguan, Aquaculture Research. 35 (2004) $436 \quad 1415-1420$.

437 2. C.R. Austin, C. Lutwak-Mann, TProc R Soc Lond B Biol Sci. 161(1964) 143-52.

438 3. S. Bhavanishankar, T. Subramoniam, J Exp Zool. 277(1997) 326-336.

439 4. E. Cabrita , V. Robles , and P. Herráez (Eds) Methods in Reproductive Aquaculture $440 \quad$ Marine and Freshwater Species, CRC Press, 2008, pp. 505-508

441 5. D. Cuccu, M. Mereu, R. Cannas, M.C. Follesa, A. Cau, P. Jereb, Journal of the 442 Marine Biological Association of the United Kingdom 87(2007) 971-976.

443 6. Development Core Team: A language and environment for statistical computing. R $444 \quad$ Foundation for statistical Computing, Vienna, Austria. (2010) ISBN 3-900051-07$445 \quad$ 0, URL http://www.R-project .org/. 
446 7. A. Diaspro, F. Beltrame, M. Fato, A. Palmeri, P. Ramoino, Microsc Res Tech. $447 \quad 36(1997) 159-64$.

448 8. P. Domingues, A. Ferreira, L. Marquez, J.P. Andrade, N. Lopez, C. Rosas, 449 Aquaculture International.16 (2008) 215-229.

450 9. A. Dunstan, CJ. Bradshaw, J. Marshall, Plos One. 10 (2011) 6.

451 10. FAO Yearbook 2010. Fishery and Aquaculture Statistics. Capture production. Rome. $452 \quad$ (2012) 609.

453 11. D.Y. Gao, J. Liu, C. Liu, L.E. McGann, , P.F. Watson, F.W. Kleinhans, P. Mazur, 454 E.S. Critser, J.K. Critser, Hum Reprod. 10 5(1995)1109-22.

455 12. D.Y. Gao, J.K. Critser, ILAR. J 414 (2000)187-96.

456 13. P. Gimenez-Bonafé, E. Ribes, P. Sautière, A. Gonzalez, H. Kasinsky, M. Kouach, 457 P.E. Sautière, J. Ausió, M. Chiva, Eur J Cell Biol. 81 (2002) 341-9.

458 14. P. Giménez-Bonafé, F.M. Soler, C. Buesa, P.E Sautière, J. Ausió, M. Kouach, H.E. 459 Kasinsky, M. Chiva, Mol Reprod Dev. 68 (2004) 232-9.

460 15. A.F. González, A.Guerra, Sarsia. 8 (1996)107-118.

461 16. J.M. Healy, Philosophical Transactions of the Royal Society of London Series B462 Biological Sciences. 323 (1989) 589-600.

463 17. J.M. Healy, Zoologica Scripta. 19 (1990) 189-193.

464 18. V. Hernández-García, Bulletin of Marine Science. 71 (2002) 347-366.

465 19. H.J.T. Hoving, V. Laptikhovsky, Biological Bulletin. 212 (1995) 177-179. 
466 20. H.J.T. Hoving, M.R. Lipinski, J.J. Videler, African Journal of rine Science. 30 $467 \quad$ (2008) 603-612.

468 21. J.T. Hoving, M.R. Lipinski, J.J. Videler, K.S.R. Bolstad, Marine Biology. 157 $469 \quad$ (2010) 393-400.

470 22. C.L. Huffard, K. Buck, B. Robison. CalCOFI Conference, San Diego, California, 471 November 26-28 Program and Abstracts, Poster-23, (2007) p 65.

472 23. J. Iglesias, F.J. Sanchez, J.G.F. Bersano, J.F. Carrasco, J. Dhont, L. Fuentes, F. 473 Linares, J.L. Munoz, S. Okumura, J. Roo, T. van der Meeren, E.A.G. Vidal, R. $474 \quad$ Villanueva. Aquaculture 266 (2007) 1-15.

475 24. Y. Ikeda, Y. Sakurai, K. Shimazaki, Invertebrate Reproduction and Development. 23 $476 \quad$ (1993) 39-44.

477 25. V.V. Laptikhovsky, C.M. Nigmatullin, Ruthenica. 6 (1996) 149-159.

478 26. R.L. Levin, J Biomech Eng. 104 (1982) 81-6.

479 27. Y.Z. Li, H. Pan, J. Xu, X.W. Fan, X.C. Song, Q. Zhang, J. Xu, Y. Liu, J Hazard $480 \quad$ Mater.179(2010) 266-75.

481 28. F. Martínez-Pastor, E. Aisen, M. Fernández-Santos, M. Esteso , A. Maroto-Morales, 482 O. García-Alvarez, J. Garde, Reproduction Cambridge England. 137 (2009) 225483235.

484 29. F. Martínez-Pastor, M.R. Fernández-Santos, E. del Olmo, A.E. Dominguez485 Rebolledo, M.C. Esteso, V. Montoro, J. Garde, Reprod Fertil Dev. 205 (2008) 547$486 \quad 556$. 
487 30. F. Martínez-Pastor, M. Mata-Campuzano, M. Álvarez-Rodríguez, M. Álvarez, L 488 .Anel, P. de Paz, Reprod Domest Anim 45 Suppl. 2 (2010) 67-78.

489

31. M.J. Naud, J.N. Havenhand, Marine Biology. 148 (2006) 559-566.

490

32. K.N. Nesis, Ruthenica 6 (1995) 23-64.

491

33. C. Nigmatullin, R.M. Sabirov, V.P. Zalygalin, Berliner Palaeobiologische 492 Abhandlungen. 3 (2003) 225-240.

493

494

495 496

39. M. Salazar, M. Lezcano, C.

C. Granja Protocol for cryopreservation of Penaeus

34. S. Nimrat, T. Sangnawakij, V. Vuthiphandchai , J. World Aquac. Soc 36 (2005) 7686.

35. G.J. Pierce, A.L. Allcock, I. Bruno, P. Bustamante, A.F. González, A. Guerra, P. Jereb, E. Lefkaditou, S.K. Malham, A. Moreno, J. Pereira, U. Piatkowski, M. Rasero, P. Sánchez, M.B. Santos, M. Santurtun, S. Seixas, I. Sobrino, R. Villanueva Cephalopod biology and fisheries in Europe. In: Anderson, E.D. (Ed.), ICES Cooperative Research Report. International Council for the Exploration of the Sea (2010) Copenhagen pp. 175.

36. V. Robles, E. Cabrita, M.P. Herráez, Zebrafish. 6 (2009) 281-93.

37. C. Rosas, J. Tut, J. Baeza, A. Sanchez, V. Sosa, C. Pascual, L. Arena, P.,Domingues, G. Cuzon. Aquaculture 275 (2008) 291-297. vannamei sperm cells in E. Cabrita, V. Robles, and P. Herráez (Eds) Methods in Reproductive Aquaculture Marine and Freshwater Species, CRC Press, 2008, pp. $505-50$. 
509 40. R. Villanueva, D. J. Staafb, J. Argüellesc, A. Bozzanoa, S. Camarillo-Coopd, et al, $510 \quad$ Aquaculture. 342-343 (2012) 125-133.

511 41. K. Watanabe, Y. Sakurai, S. Segawa, T. Okutani, American Malacological Bulletin. 13 $512 \quad$ (1996) 73-88.

513

514

515 


\section{7. Figure captions}

519 Figure 1. Illex coindetii spermatophores from the spermatophoric organ (Needham's

520 sac) of a mature male (left), and a bulb of spermatangia from a copulated female (right).

521 Scale bar: $2 \mathrm{~mm}$.

523 Figure 2. Scanning electron microscope images of Illex coindetii spermatozoa obtained

524 from spermatangia, showing the whole spermatozoon with two tails (top), the head

525 region with the annular atria (centre) and acrosomal region (bottom). Scale bar: $3 \mu \mathrm{m}$.

527 Figure 3. Spermatozoa plasma membrane status (intact, increased permeability and 528 damaged) and mitochondrial status after subjecting the Illex coindetii spermatophores to $5295 \%$ or $15 \%$ of different permeating cryoprotectants (ETG: ethylene glycol, GLY: 530 glycerol, MET: methanol, PPD: 1,2-propanediol) for $5 \mathrm{~min}$, or to medium without 531 cryoprotectant (Control). Medians in each treatment are indicated by vertical lines.

532 When only significant main effects were detected, they are indicated in the inset text. If 533 interactions between cryoprotectant type and concentration were significant, we carried 534 out a pairwise comparison among treatments. In this case, lines join significantly 535 different treatments ( $\mathrm{P}$ value showed). Active mitochondria ratio is the proportion of 536 spermatozoa with active mitochondria within the intact membrane population.

538 Figure 4. Time dynamics of membrane and mitochondrial status of Illex coindetii 539 spermatozoa (mean \pm SEM at 5, 15 and $30 \mathrm{~min}$ ), after submitting the spermatophores to $54015 \%$ of the cryoprotectants Me2SO, ethylene glycol (ETG), glycerol (GLY), methanol 
541 (MET) or 1,2-propanediol (PPD). When only significant main effects were detected, 542 they are indicated in the inset text. If interactions between cryoprotectant type and 543 incubation time were significant, we carried out a pairwise comparison among 544 treatments within each time. In this case, different letters indicate incubation times when 545 treatments were significantly different (differences detailed in the inset text). Active 546 mitochondria ratio is the proportion of spermatozoa with active mitochondria within the 547 intact membrane population.

548 
Figure 1
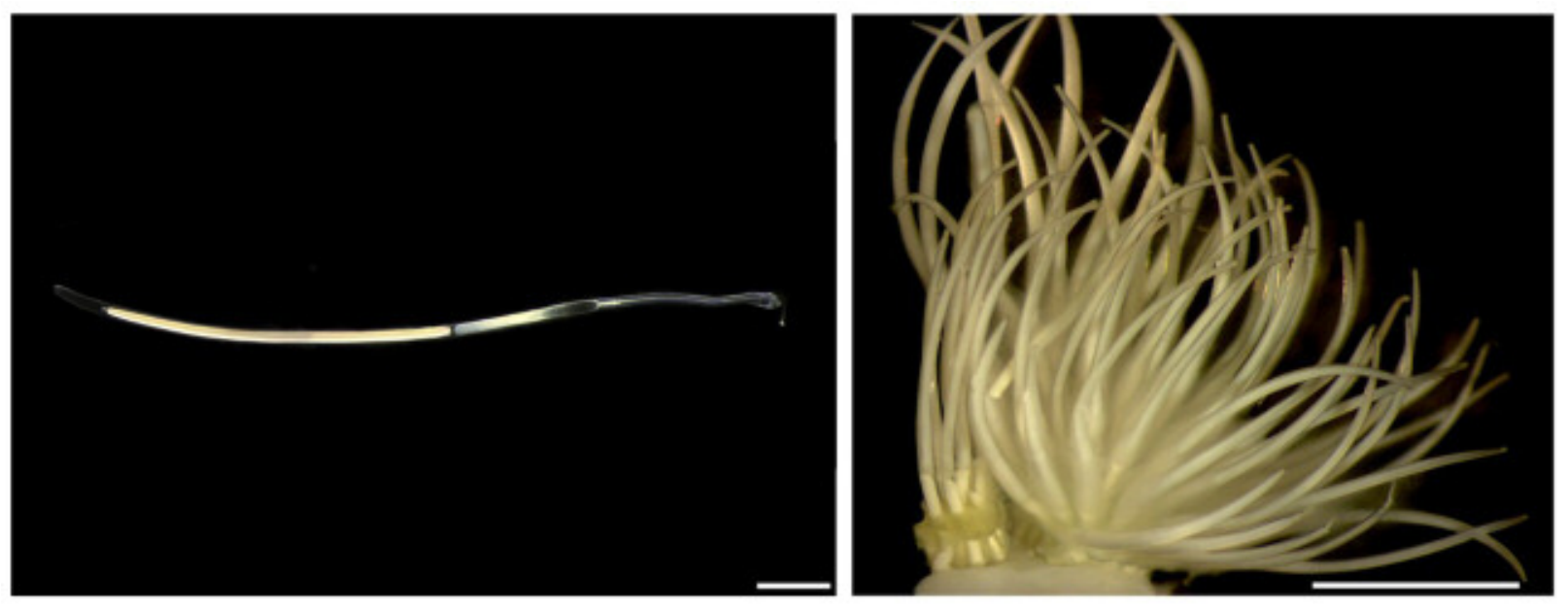

Figure(s) 
Figure 2
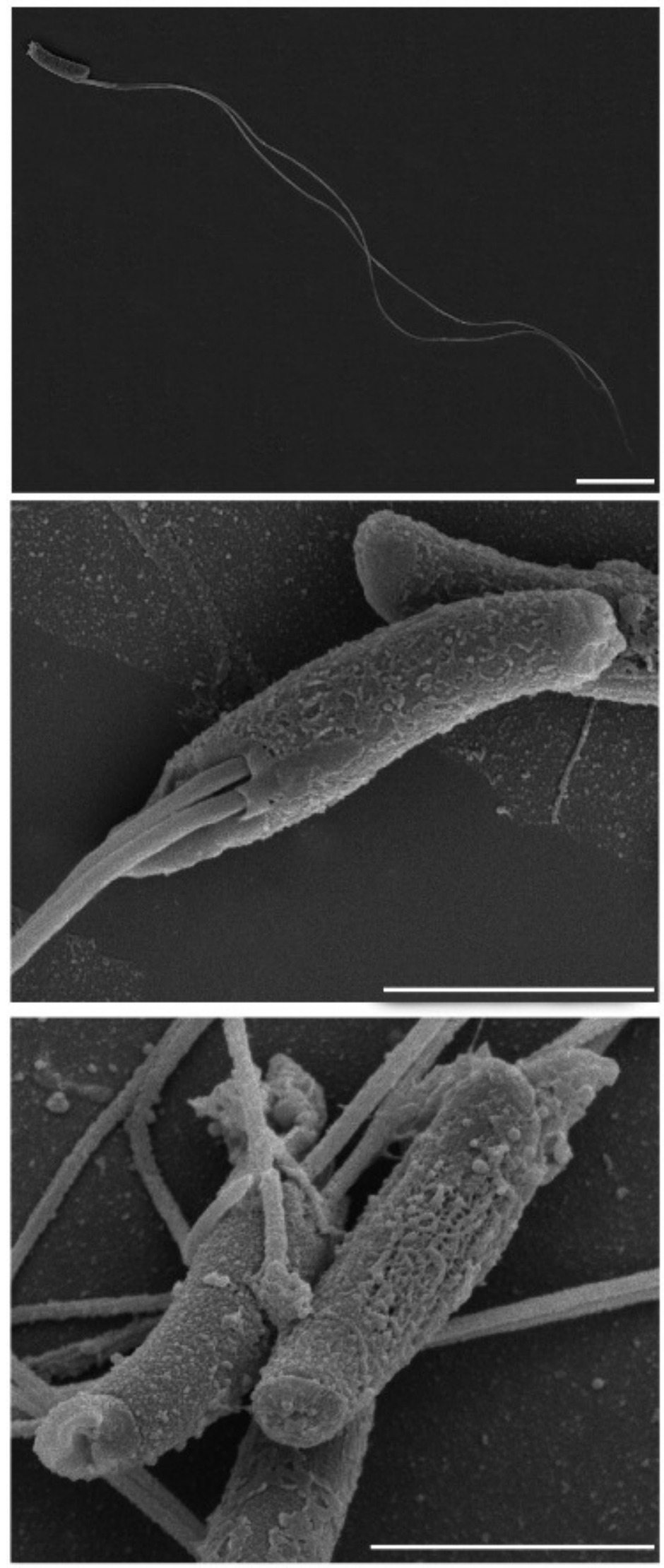


\section{Figure 3}

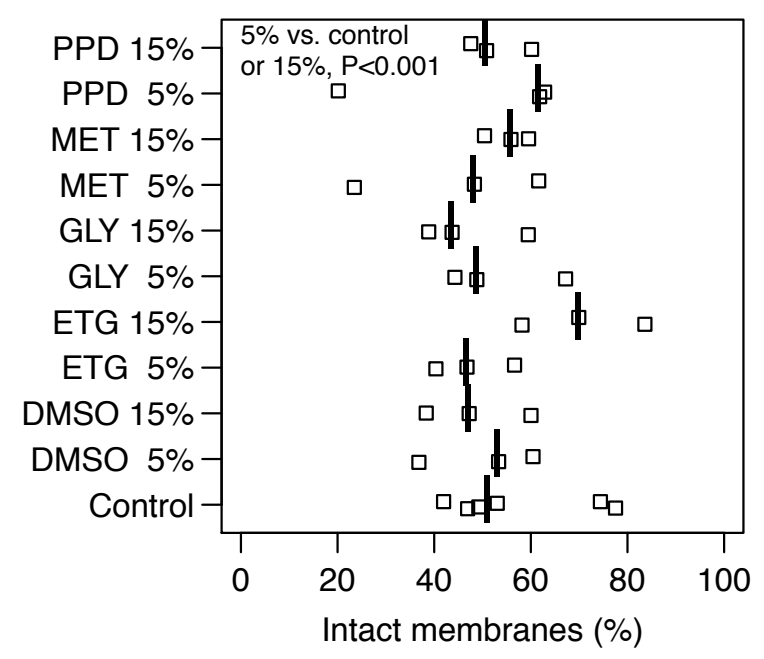

(a) Viability (YO-PRO-1-/H342-)

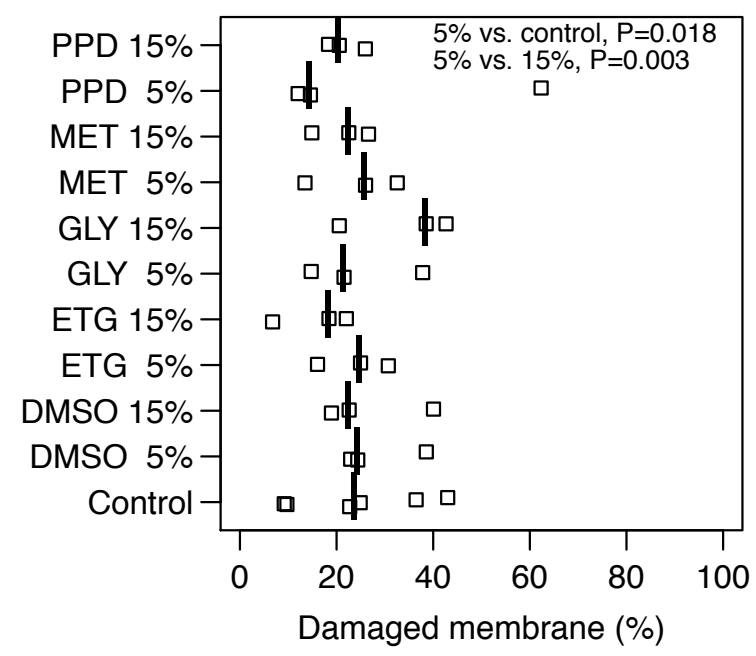

(c) Damaged membrane (YO-PRO-1+/H342+)

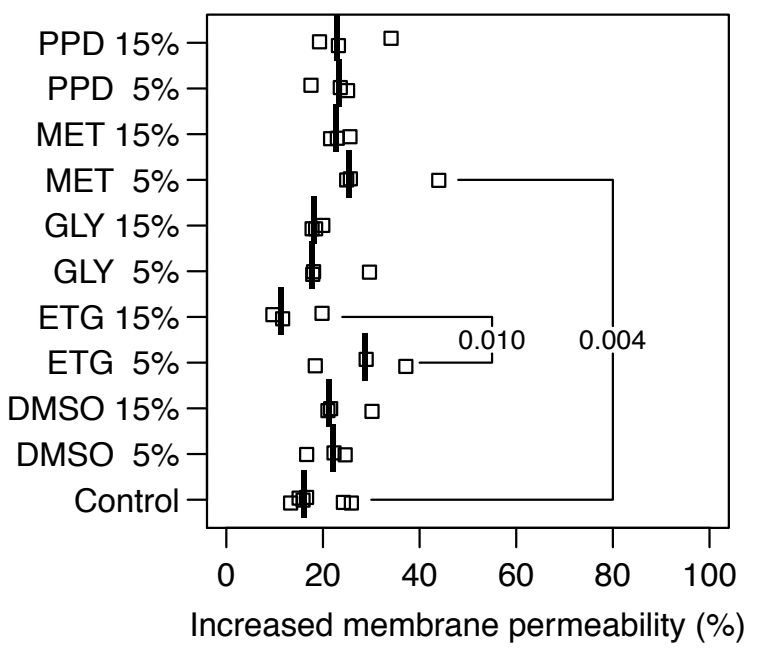

(b) Membrane permeability (YO-PRO-1+/H342-)

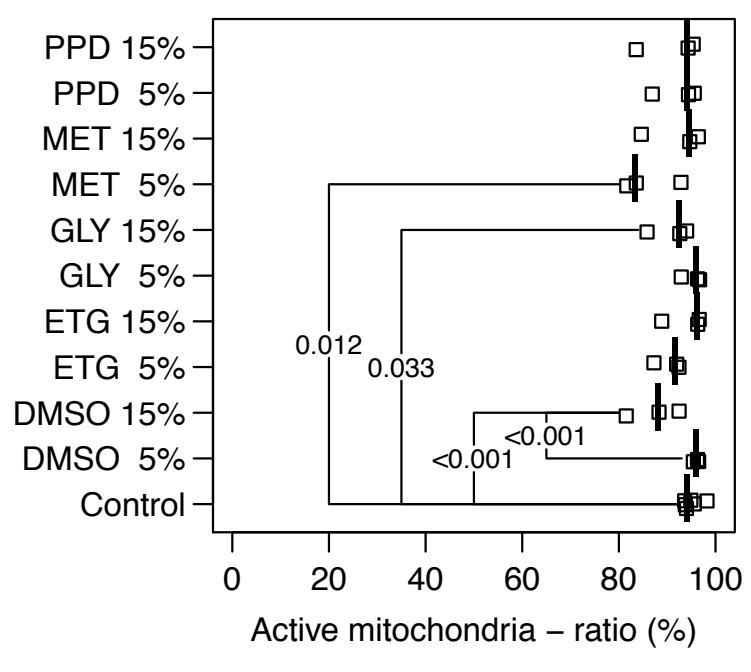

(d) Active mitochondria (Mitotracker+) 


\section{Figure 4}
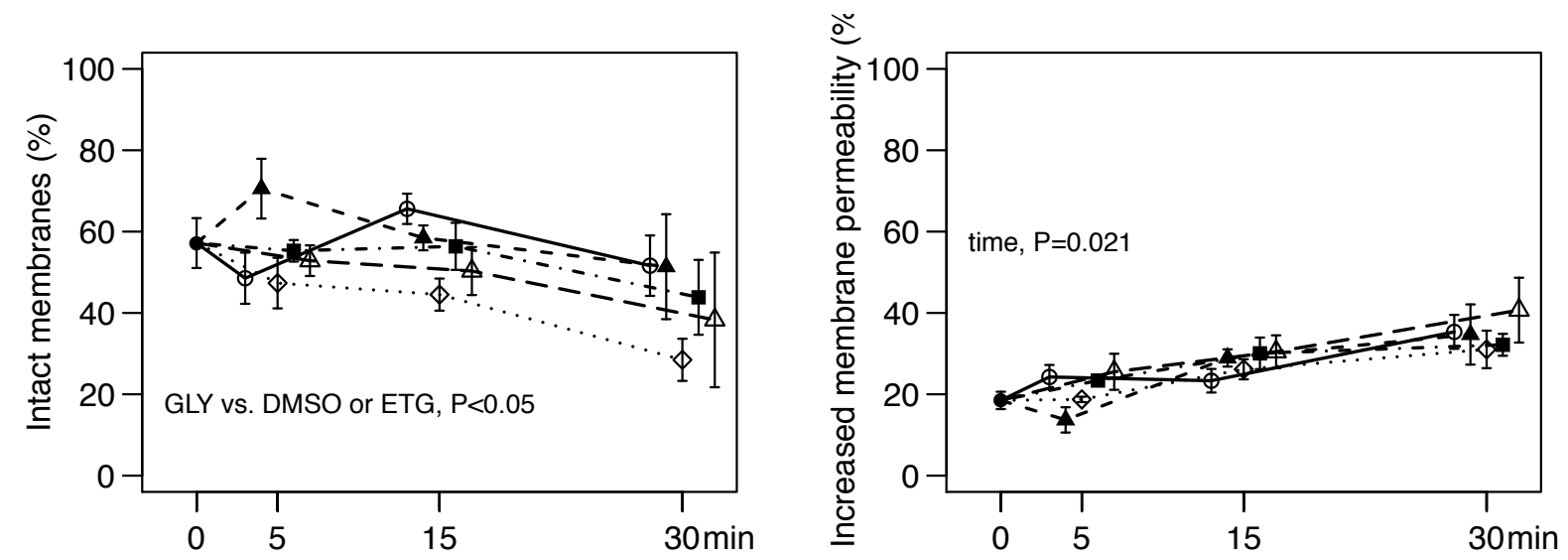

(a) Viability (YO-PRO-1-/H342-)

(b) Membrane permeability (YO-PRO-1+/H342-)
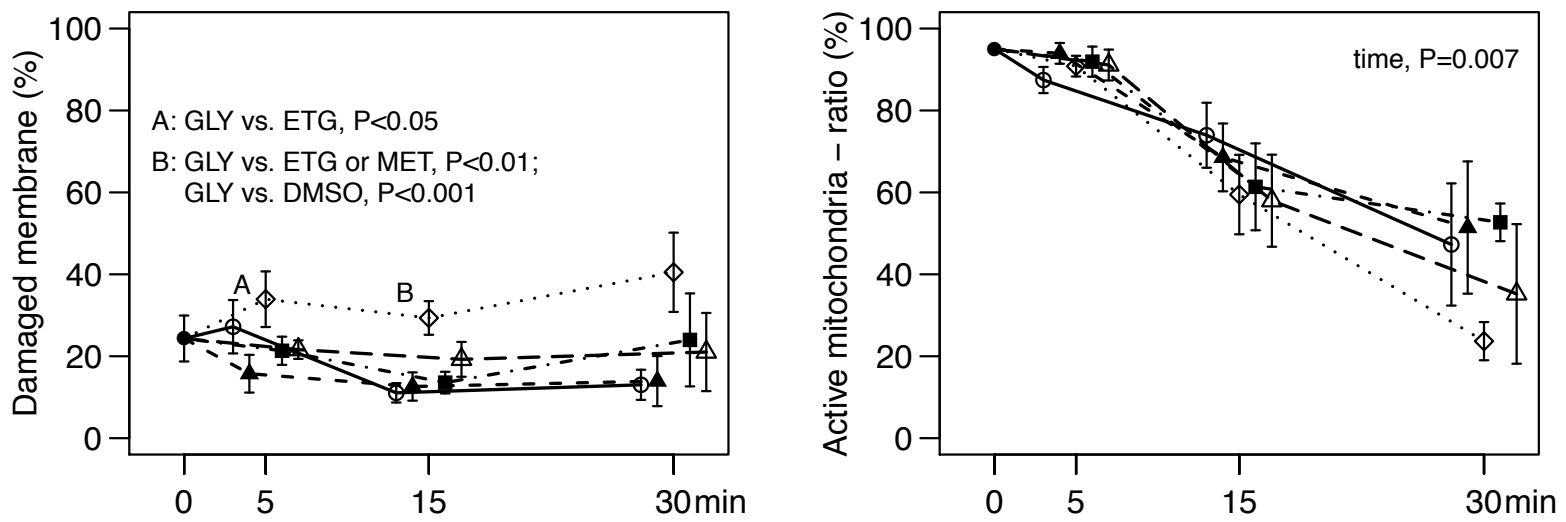

(c) Damaged membrane (YO-PRO-1+/H342+)

(d) Active mitochondria (Mitotracker+)

- CTL $\bigcirc$ DMSO - $\mathbf{\Delta}$ - ETG 
This work has been funded by the Spanish Ministry of Science and Innovation (MICINN) research projects AGL2009-11546 and AGL2009-06994 and Fundación Ramón Areces. 